\title{
THE IMPORTANCE OF COMPRESSION SONOELASTOGRAPHY IN IMPROVING THE DIAGNOSTICS OF THE PATHOLOGY OF MYOMETRIUM
}

\author{
Olena Sharmazanova \\ Department of Radiation Diagnostics ${ }^{1}$ \\ Inessa Safonova \\ Department of Radiation Diagnostics ${ }^{1}$ \\ Yulia Mityakova $\bowtie$ \\ Department of Radiation Diagnostics ${ }^{1}$ \\ diagnost-uzi@ukr.net \\ ${ }^{1}$ Kharkiv Medical Academy of Postgraduate Education \\ 58 Amosova str., Kharkiv, Ukraine, 61176
}

$\triangle$ Corresponding author

Abstract

The diversity of the clinical picture and the asymptomatic nature of the clinical manifestations of myometrial pathology cause difficulties in diagnosis. There is a lack of reliable diagnostic criteria for this pathology, in particular, imaging, especially with the simultaneous combination of adenomyosis and leiomyoma.

The aim of the research. Determination of the possibility of compression sonoelastography in the diagnosis of myometrial pathology and determination of its sonoelastography characteristics in leiomyoma and adenomyosis, as well as comparison of sonoelastography results with histological data.

Materials and methods. Elastography images of 155 patients with adenomyosis and leiomyoma, as well as combined pathology, were analyzed, the elastography diagnosis of which was confirmed by histological examination.

Results. Leiomyoma and adenomyosis had different elastography characteristics (strain ratios) with different color mapping; their specific characteristics and main differences are determined. Based on sonoelastography, the majority of patients $(n=30)$ were suspected of having uterine fibroids, 14 had adenomyosis, and 42 had adenomyosis and fibroids. Sonoelastography revealed histological signs of adenomyosis in 3 patients with uterine leiomyoma.

Conclusions. Ultrasound examination using compression sonoelastography in such pathological conditions of the myometrium as adenomyosis and leiomyoma, as well as unchanged myometrium, makes it possible to determine changes in the degree of elasticity of the myometrium in the corresponding pathology. Sonoelastography allows the identification of clear distinguishing features of fibroids and adenomyosis. The unchanged myometrium has a certain elasticity, which can be equated to a specific numerical value - the coefficient of deformation. This indicator has different meanings in myoma and adenomyosis, which makes it possible to differentiate these pathological conditions of the myometrium. Compression sonoelastography is able to identify clear distinguishing features of leiomyoma and adenomyosis, and consistency of diagnoses based on sonoelastography and histology is significant but not optimal.

Keywords: adenomyosis, leiomyoma, compression sonoelastography, uterus, myometrium, ultrasound, study, image, deformation ratio, density.

DOI: $10.21303 / 2504-5679.2021 .001908$

\section{Introduction}

Adenomyosis remains an urgent problem in medicine due to an increase in morbidity, diagnostic difficulties and a lack of unambiguity in understanding its pathogenesis. The prevalence of adenomyosis ranges from 5 to $70 \%$ [1]. By the age of 40, the disease affects 2 out of 10 women, and at the age of 40 to 50 , the incidence increases: approximately 8 out of 10 women have this pathology [2, 3]. However, the incidence of adenomyosis is difficult to establish due to the lack of a single definition and diagnostic criteria based on non-invasive diagnostic tests [4]. Crucial in the diagnosis of adenomyosis, the identification of its frequency and structural and functional features, as before, belongs to the histological examination of the removed uterus [5]. In one third of cases, 
the course of adenomyosis is asymptomatic [6,7]. Frequent clinical symptoms of adenomyosis are menorrhagia (up to $50 \%$ of patients), dysmenorrhea (30\%) and metrorrhagia (20\%); it also often accompanies the processes of uterine enlargement and infertility [2, 5].

According to the scientific literature, the most common benign gynecological tumour in women of reproductive age is uterine leiomyoma [8]. Unfortunately, this pathology is detected in 20-30\% of women of reproductive age [9], recently there is a process of «rejuvenation» of the disease, in $20-25 \%$ of women with leiomyoma, and complete infertility is observed [10]. Therefore, the problem of diagnosis and treatment of leiomyoma is urgent.

Adenomyosis can be combined with estrogen-dependent benign diseases: endometriosis $(70 \%)$, uterine leiomyoma (50\%), endometrial hyperplasia (35\%) [5]. M. Genc et al. after conducting a retrospective analysis of patients after hysterectomy, found a positive correlation between the progression of adenomyosis and previous abortions and pregnancies, the presence of fibroids. However, the researchers found no correlation with smoking, normal childbirth, caesarean section, endometrial hyperplasia and ovarian endometriosis [11].

Difficulties in diagnosing myometrial pathology lie in the diversity of the clinical picture and the asymptomatic nature of clinical manifestations. There is a lack of reliable diagnostic criteria for this pathology, in particular, histological and imaging, especially if the same patient has a simultaneous combination of adenomyosis and leiomyoma $[12,13]$.

The main method of instrumental diagnostics, which determines further therapeutic tactics, is ultrasound. It should be noted the possibilities of ultrasound in the diagnosis of uterine fibroids, which allow a detailed assessment of the features of the localization of nodes, their echostructure, hemodynamics and enlargement [14]. New developments have made it possible to supplement the information of ultrasound examination with 3D and 4D images [15]. However, it should be noted that the frequency of diagnostic errors in the detection of adenomyosis and uterine leiomyoma is still quite high.

It is noteworthy that the data presented in the literature on the informativeness of ultrasound in detecting adenomyosis and uterine fibroids are rather contradictory. For example, the accuracy of the diagnosis of adenomyosis varies, according to the literature, in the range from 38.4 to $86.4 \%$ [16]. The data on the sensitivity of ultrasound in the diagnosis of uterine fibroids vary from $69 \%$ [17] to $78.1 \%$, which is associated with a decrease in the information content of the method in the case of an increase in the number of fibroid nodes [18].

The technique of sonoelastography, an imaging technology, which makes it possible to qualitatively and quantitatively assess the physical properties of tissues, their elasticity and density, is becoming more and more widespread. Now this technique is used as an additional one during differential diagnostics to identify pathological changes in tissues. The sonoelastography technique makes it possible to assess the density of the myometrium, which is important in determining the predominant process in mixed forms of leiomyoma and adenomyosis to determine the treatment plan.

The aim of the study was to determine the possibility of compression sonoelastography in the diagnosis of myometrial pathology and to determine its sonoelastography characteristics in leiomyoma and adenomyosis, as well as to compare the results of sonoelastography with histological data.

\section{Materials and methods}

The work is based on the analysis of the results of the study of the elastography characteristics of the uterus, which were obtained as a result of transvaginal examination using compression sonoelastography.

During compression sonoelastography imaging, the ultrasound machine detects tissue displacement by tracking ultrasonic spots and comparing conditions before and after applying pressure. The warp change is linked to colour and superimposed on the corresponding B-mode image. The advantage of this method is the ability to simultaneously acquire a two-dimensional (2D) ultrasound image and an elastography image using a pressure transducer. An additional advantage of the method is the comparative ease of image interpretation.

We examined 155 women who were examined on the basis of the diagnostic ultrasound centre «Diagnost» in the period from September 2017 to December 2020. The average age of the studied patients was $44 \pm 3.6$ years. 
In the course of the study, the rules for ensuring the safety of patients' health, their rights, human dignity, moral and ethical standards were observed in accordance with the requirements of the Declaration of Helsinki of the World Medical Association «Ethical principles of medical research with human participation as an object of research» (as amended on 01.10.2008 G.). The patients who participated in the study were informed in detail about its purpose, objectives and gave informed consent to participate in it, as well as the processing of personal data (Ukraine, KhMAPE, protocol No. 36 of 10.12.2018).

All patients underwent a complex ultrasound scan using a HITACHI AVIUS apparatus (Hitachi Medical Corporation, Japan) with a supporting sonoelastography function. At the beginning, all patients underwent transvaginal ultrasound examination (TVUSE) using an endocavital transducer with a frequency of 8-4 MHz. During the study, the cervix and body of the uterus, ovaries and fallopian tubes were sequentially visualized, the position of the body of the uterus, its shape, contours and dimensions were assessed. To clarify the nature of the detected pathological process of the myometrium, all patients underwent compression sonoelastography using the same ultrasound apparatus.

Group 1 included 30 women with leiomyoma, group 2 consisted of 14 women with suspected adenomyosis, the criterion for inclusion in group 3 (42 women) was the presence of combined pathology of adenomyosis and leiomyoma. The average age of the patients is 38 years.

Elastograms were evaluated using a computerized colour scale, on which the degree of rigidity (deformation) corresponded to a certain colour: soft tissues - red and green, hard - blue. The coefficient of deformation was determined according to the standard point scale of stiffness.

At the second stage of the study, to assess the specific elastography characteristics of leiomyoma and adenomyosis, we analyzed elastography images of adenomyosis and images of leiomyoma in those patients in whom the elastography diagnosis was confirmed by histological examination. We compared the colour indices of leiomyoma and adenomyosis using Fisher's exact test.

The quantitative values of the deformation coefficient were determined automatically at the study site: average value (Emean), minimum value (Emin), maximum value (Emax). Emin, Emax and SD were used for analysis, after which Emean was determined.

Statistical analysis was performed using the MedCalc statistical software, statistical data processing was performed by the variational statistical method using correlation analysis and Student's t test. The difference between the series of variations was considered probable at $p<0.05$.

86 women underwent elastography imaging. Histological examination was performed on 86 patients, all of whom also managed to obtain a stable elastography image. Baseline characteristics of all patients with histology results are presented in Table $\mathbf{1 .}$

Table 1

Characteristics of the patients included in the study $(n=86)$

\begin{tabular}{ccc}
\hline Patient characteristics & Quantitative indicator & $\boldsymbol{p}$ \\
\hline & Histological diagnosis & $<0.001$ \\
\hline Adenomyosis & $2.4-6.0$ & $<0.001$ \\
Leiomyoma & $0.7-1.5$ & $<0.001$ \\
\hline Adenomyosis + leiomyoma & $0.9-3.5$ & $<0.001$ \\
\hline Laparoscopic hysterectomy & Type of surgery & $<0.001$ \\
Vaginal hysterectomy & 34 & $<0.001$
\end{tabular}

\section{Results}

Retained dynamic stable images obtained as a result of assessment of elastography characteristics of the uterus and intrauterine abnormalities, which were confirmed histologically $(n=86)$, were analyzed. In most cases, the myometrium and the serous membrane of the uterus 
had a uniform appearance on the elastography image, stained with a red colour characteristic of the soft-elastic structure. The uterus was well separated from the surrounding intestine as shown by several small, bright, parallel lines. The average number of coloured lines was 4.3 (SD 1.6, range $2-13)$. In most cases $(95 \%)$, the serous membrane began with a green line followed by a transition to a red line (Fig. 1).

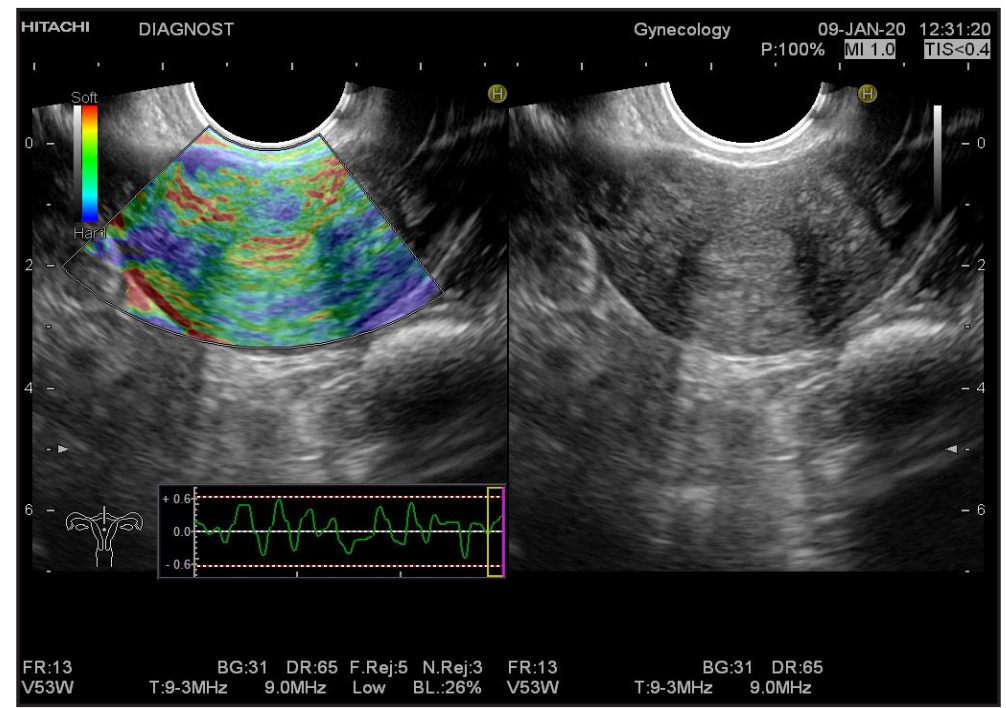

Fig. 1. The use of compression sonoelastography with unaltered myometrium and intact serous membrane of the uterus (shown as a red line)

In $79 \%$ of cases, the myometrium had a uniform coloration, the main colour ranged from green to blue-green coloration.

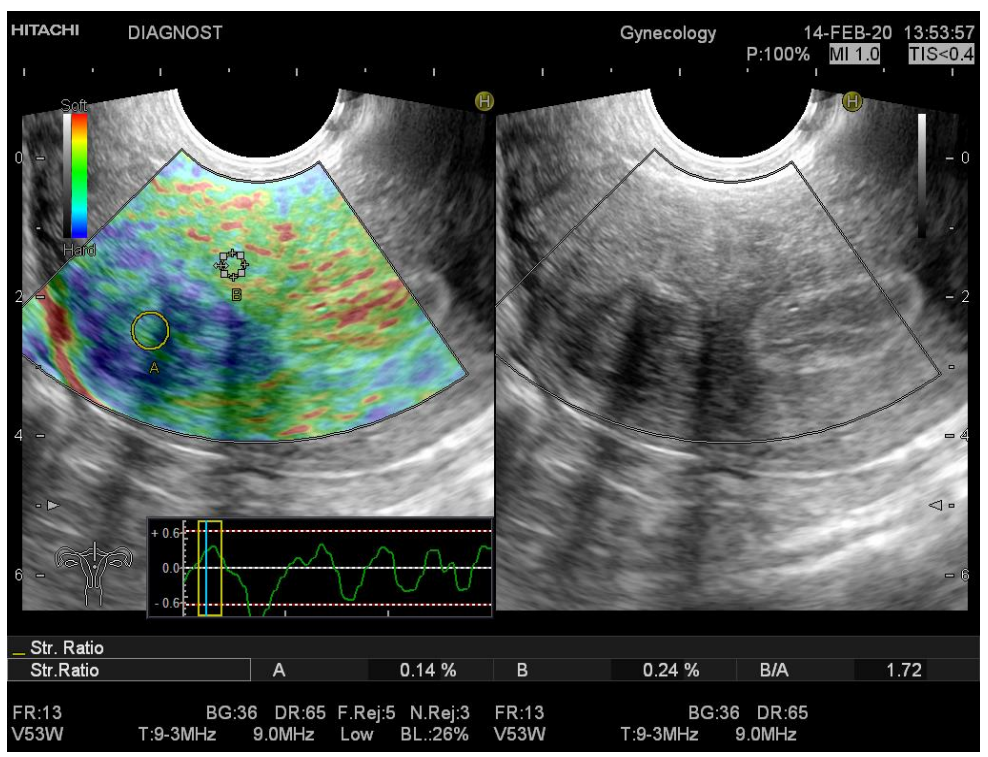

Fig. 2. Leiomyoma in a 42-year-old patient. On the right is the B-mode image.

Left - image using compression sonoelastography. Index B - control zone.

Pointer A - leiomyoma (predominantly blue)

Leiomyoma and adenomyosis had different elastography characteristics (strain ratios) with different colour mapping. Examples of elastography images of fibroids are shown in Fig. 2, and the image of adenomyosis is in Fig. 3. In general, leiomyomas were mapped in blue, darker than the adjacent myometrium, and adenomyosis, in yellow-green (more elastic) than the adjacent 
myometrium. Most leiomyomas had a regular shape with a clear capsule, while adenomyosis was predominantly irregular in shape without clear boundaries and capsules. The fibroid capsule was mapped predominantly in red and was traced throughout. In adenomyosis, clear boundaries were absent with a smooth transition to the myometrium with replaceable mapping from yellow-green to green-blue.

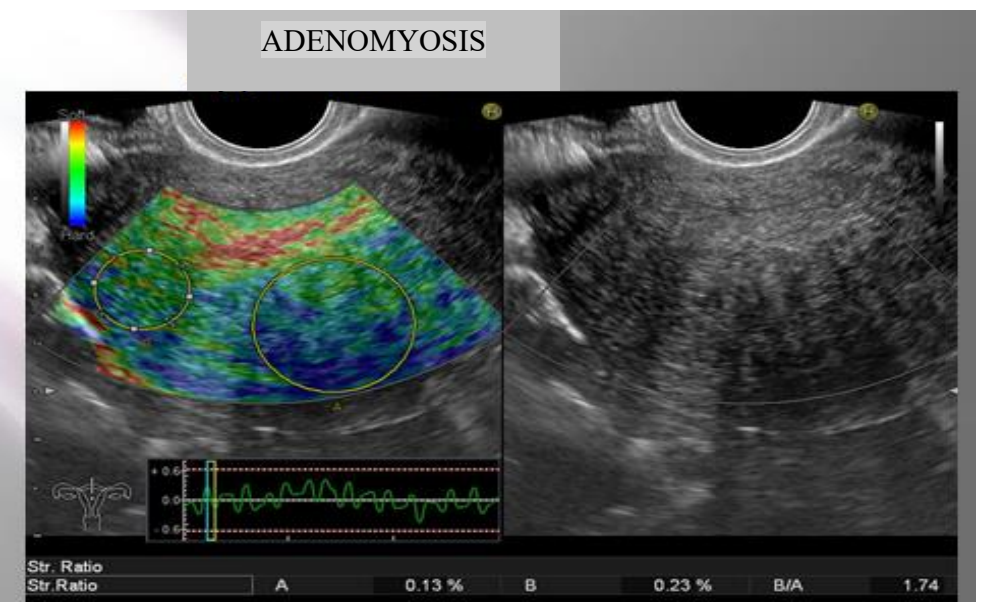

Fig. 3. Compression sonoelastography image of a 38-year-old patient with adenomyosis.

The defeat of the predominantly posterior wall with a predominance of blue, without visible clear boundaries. $\mathrm{CD}=1.74$

Specific characteristics and main differences between fibroids and adenomyosis are presented in Table 2.

Table 2

Elastography characteristics of uterine fibroids and adenomyosis based on histological diagnosis

\begin{tabular}{|c|c|c|c|c|}
\hline \multirow{2}{*}{ Characteristics } & \multicolumn{2}{|c|}{ Histological diagnosis } & \multicolumn{2}{|c|}{ Elastography characteristics } \\
\hline & Leiomyoma $(n=30)$ & Adenomyosis $(n=14)$ & Leiomyoma $(n=30)$ & Adenomyosis $(n=14)$ \\
\hline Uniform shape & 30 & 14 & Uniform shape & Wrong form \\
\hline$>50 \%$ uniform color & 28 & 12 & $>50 \%$ uniform color & $<50 \%$ uniform color \\
\hline $\begin{array}{l}\text { Center color compared to } \\
\text { myometrium: } \\
\text { - darker; } \\
\text { - same; } \\
\text { - lighter }\end{array}$ & $\begin{array}{l}\text { Darker in relation to } \\
\text { the myometrium }\end{array}$ & $\begin{array}{l}\text { Equal or lighter } \\
\text { in relation to the } \\
\text { myometrium }\end{array}$ & $\begin{array}{l}\text { The center is predomi- } \\
\text { nantly darker or the } \\
\text { same as the myometrium }\end{array}$ & $\begin{array}{l}\text { The center is lighter } \\
\text { than the myometrium }\end{array}$ \\
\hline Focal lesion capsule & Red & Green & The capsule is clear, red & $\begin{array}{l}\text { The border is fuzzy. } \\
\text { Without capsule with } \\
\text { smooth transition }\end{array}$ \\
\hline Clear border/capsule & Clear & Not clear & Visible capsule in most & $\begin{array}{l}\text { Minority's visible } \\
\text { border }\end{array}$ \\
\hline Regular capsule & Without features & $\begin{array}{l}\text { Drainage. } \\
\text { Not uniform }\end{array}$ & Normal capsule at $90 \%$ & Jagged border \\
\hline Number of lines per capsule & - & - & Capsule $\geq 5$ lines & Border 1-2 lines \\
\hline
\end{tabular}

Based on sonoelastography, the majority of patients $(n=30)$ were suspected of having uterine fibroids, 14 had adenomyosis, and 42 had adenomyosis and fibroids. Table 3 shows the data of the agreement of diagnoses based on sonoelastography and histology. Sonoelastography revealed histological signs of adenomyosis in 3 patients with uterine fibroids. 
Table 3

Reconciliation of diagnoses based on sonoelastography and histology data

\begin{tabular}{cccc}
\hline \multirow{2}{*}{ Diagnosis based on sonoelastography data } & \multicolumn{3}{c}{ Histological diagnosis } \\
\cline { 2 - 4 } & Leiomyoma & Adenomyosis & Leiomyoma and adenomyosis \\
\hline Leiomyoma & 30 & - & - \\
Adenomyosis & - & 14 & 45
\end{tabular}

\section{Discussion}

Normal myometrium, endometrium, leiomyoma, and adenomyosis are well visualized with sonoelastography. A standardized compression sonoelastography imaging technique was defined and applied for the diagnosis of leiomyoma and adenomyosis. Certain patterns and features, taking into account the shape and colour mapping, can be recognized, they can be used to identify these pathologies and distinguish them from the tissues of the unchanged uterus. A significant agreement was found between the diagnosis of leiomyoma and adenomyosis based on compression sonoelastography and histological diagnosis.

In the literature, there are studies on the use of compression elastography in the diagnosis of myometrial pathology $[19,20]$, although there are quite a few of them. The characteristics of fibroids and adenomyosis identified in our study are consistent with these studies.

In this study, some leiomyomas had the same colour intensity as the adjacent myometrium, and $65 \%$ were darker in colour. According to B. Stoelinga et al. [20], the proportion of patients whose main myometrium colour was purple or dark blue, which indicated a harder tissue, was $49 \%$. This can be attributed to differences in the pressure force of the transducer to acquire the image, as well as differences in the devices used.

Due to the fact that the image obtained by compression sonoelastography is influenced by the amount of applied pressure, some experience is required to obtain high-quality reproducible images, as well as their interpretation. The difficulty of mastering the method of sonoelastography lies mainly in the fact that different patients vary the mobility of the uterus and have their own physiological characteristics. In addition, the pressure of the transducer applied to the cervix and the frequency of the specialist's movements are different, often resulting in images that are difficult to reproduce. As you gain experience, it becomes easier to get an image. We examined about 86 patients for elastography imaging in order to develop a standardized imaging technique and learn how to interpret the images. A detailed description of specific signs of leiomyoma and adenomyosis in images of a dynamic state revealed significant agreement with histology data and can be used in future studies that will evaluate the diagnostic accuracy of the compression sonoelastography method and its additional value compared to conventional seroscale ultrasound with / without Doppler function.

Study limitations. The study had some limitations due to the small sample size of patients.

Prospects for further researches consist in the development of prognostic indicators of the deformation coefficient in ultrasound diagnostics using compression sonoelastography to assess the effectiveness of therapeutic measures and dynamic observation of patients with myometrial pathology.

\section{Conclusions}

Ultrasound examination using compression sonoelastography in such pathological conditions of the myometrium as adenomyosis and leiomyoma, as well as unchanged myometrium, makes it possible to determine changes in the degree of elasticity of the myometrium in the corresponding pathology. Sonoelastography allows the identification of clear distinguishing features of fibroids and adenomyosis. The unchanged myometrium has a certain elasticity, which can be equated to a specific numerical value - the coefficient of deformation. This indicator has different meanings in myoma and adenomyosis, which makes it possible to differentiate these pathological conditions of the myometrium. 
Thus, compression sonoelastography is able to identify clear distinguishing features of leiomyoma and adenomyosis, and the consistency of diagnoses based on sonoelastography and histology is significant, but not optimal.

\section{Conflict of interests}

The authors declare that they have no conflicts of interest.

\section{Financing}

The study was performed without financial support.

\section{References}

[1] Graziano, A., Lo Monte, G., Piva, I., Caserta, D., Karner, M., Engl, B., Marci, R. (2015). Diagnostic findings in adenomyosis: a pictorial review on the major concerns. European review for medical and pharmacological sciences, 19 (7), $1146-1154$.

[2] Harada, T., Khine, Y. M., Kaponis, A., Nikellis, T., Decavalas, G., Taniguchi, F. (2016). The Impact of Adenomyosis on Women's Fertility. Obstetrical \& gynecological survey, 71 (9), 557-568. doi: http://doi.org/10.1097/ogx.0000000000000346

[3] Moroni, R. M., Vieira, C. S., Ferriani, R. A., dos Reis, R. M., Nogueira, A. A., Brito, L. G. O. (2015). Presentation and treatment of uterine leiomyoma in adolescence: a systematic review. BMC Women's Health, 15 (1). doi: http://doi.org/10.1186/ s12905-015-0162-9

[4] Li, J.-J., Chung, J. P. W., Wang, S., Li, T.-C., Duan, H. (2018). The Investigation and Management of Adenomyosis in Women Who Wish to Improve or Preserve Fertility. BioMed Research International, 2018, 1-12. doi: http://doi.org/10.1155/2018/6832685

[5] Eisenberg, V. H., Arbib, N., Schiff, E., Goldenberg, M., Seidman, D. S., Soriano, D. (2017). Sonographic Signs of Adenomyosis Are Prevalent in Women Undergoing Surgery for Endometriosis and May Suggest a Higher Risk of Infertility. BioMed Research International, 2017, 1-9. doi: http://doi.org/10.1155/2017/8967803

[6] Abbott, J. A. (2017). Adenomyosis and Abnormal Uterine Bleeding (AUB-A) - Pathogenesis, diagnosis, and management. Best Practice \& Research Clinical Obstetrics \& Gynaecology, 40, 68-81. doi: http://doi.org/10.1016/j.bpobgyn.2016.09.006

[7] Mas, A., Tarazona, M., Dasí Carrasco, J., Estaca, G., Cristóbal, I., Monleón, J. (2017). Updated approaches for management of uterine fibroids. International Journal of Women's Health, Volume 9, 607-617. doi: http://doi.org/10.2147/ijwh.s138982

[8] Al-Hendy, A., Myers, E., Stewart, E. (2017). Uterine Fibroids: Burden and Unmet Medical Need. Seminars in Reproductive Medicine, 35 (6), 473-480. doi: http://doi.org/10.1055/s-0037-1607264

[9] Avramenko, N. V., Barkovsky, D. E., Kabachenko, O. V., Letsin, D. V. (2017). Reproductologist's current views on etiopathogenesis and treatment of uterine leiomyoma. Zaporozhye Medical Journal, 3 (19), 381-386. doi: http://doi.org/10.14739/ 2310-1210.2017.3.100953

[10] Lisiecki, M., Paszkowski, M., Woźniak, S. (2017). Fertility impairment associated with uterine fibroids - a review of literature. Menopausal Review, 16 (4), 137-140. doi: http://doi.org/10.5114/pm.2017.72759

[11] Genc, M., Genc, B., Cengiz, H. (2014). Adenomyosis and accompanying gynecological pathologies. Archives of Gynecology and Obstetrics, 291 (4), 877-881. doi: http://doi.org/10.1007/s00404-014-3498-8

[12] Chapron, C., Vannuccini, S., Santulli, P., Abrão, M. S., Carmona, F., Fraser, I. S. et. al. (2020). Diagnosing adenomyosis: an integrated clinical and imaging approach. Human Reproduction Update, 26 (3), 392-411. doi: http://doi.org/10.1093/ humupd/dmz049

[13] Naftalin, J., Hoo, W., Nunes, N., Holland, T., Mavrelos, D., Jurkovic, D. (2016). Association between ultrasound features of adenomyosis and severity of menstrual pain. Ultrasound in Obstetrics \& Gynecology, 47 (6), 779-783. doi: http://doi.org/ 10.1002/uog.15798

[14] Mitkov, V. V., Khuako, S. A., Tsyganov, S. E., Kirillova, T. A., Mitkova, M. D. (2013). Comparative Analysis of Shear Wave Elastography and Results of Uterine Morphological Examination (Preliminary Results). Ultrasound and Functional Diagnostics, 5, 99-114.

[15] Zhang, M., Wasnik, A. P., Masch, W. R., Rubin, J. M., Carlos, R. C., Quint, E. H., Maturen, K. E. (2018). Transvaginal Ultrasound Shear Wave Elastography for the Evaluation of Benign Uterine Pathologies: A Prospective Pilot Study. Journal of Ultrasound in Medicine, 38 (1), 149-155. doi: http://doi.org/10.1002/jum.14676

[16] Guerriero, S., Saba, L., Pascual, M. A., Ajossa, S., Rodriguez, I., Mais, V., Alcazar, J. L. (2018). Transvaginal ultrasound vs magnetic resonance imaging for diagnosing deep infiltrating endometriosis: systematic review and meta-analysis. Ultrasound in Obstetrics \& Gynecology, 51 (5), 586-595. doi: http://doi.org/10.1002/uog.18961

[17] Karageyim Karsidag, A. Y., Buyukbayrak, E. E., Kars, B., Unal, O., Turan, M. C. (2009). Transvaginal sonography, sonohysterography, and hysteroscopy for investigation of focal intrauterine lesions in women with recurrent postmenopausal 
bleeding after dilatation \& curettage. Archives of Gynecology and Obstetrics, 281 (4), 637-643. doi: http://doi.org/10.1007/ s00404-009-1150-9

[18] Wanderley, M., Álvares, M., Vogt, M., Sazaki, L. (2016). Accuracy of Transvaginal Ultrasonography, Hysteroscopy and Uterine Curettage in Evaluating Endometrial Pathologies. Revista Brasileira de Ginecologia e Obstetrícia / RBGO Gynecology and Obstetrics, 38 (10), 506-511. doi: http://doi.org/10.1055/s-0036-1593774

[19] Stoelinga, B., Hehenkamp, W. J. K., Brölmann, H. A. M., Huirne, J. A. F. (2014). Real-time elastography for assessment of uterine disorders. Ultrasound in Obstetrics \& Gynecology, 43 (2), 218-226. doi: http://doi.org/10.1002/uog.12519

[20] Stoelinga, B., Hehenkamp, W., Nieuwenhuis, L. L., Conijn, M., van Waesberghe, J., Brölmann, H., Huirne, J. (2018). Accuracy and Reproducibility of Sonoelastography for the Assessment of Fibroids and Adenomyosis, with Magnetic Resonance Imaging as Reference Standard. Ultrasound in medicine \& biology, 44 (8), 1654-1663.doi: https://doi:10.1016/j.ultrasmedbio.2018.03.027

Received date 24.06.2021

(C) The Author(s) 2021

Accepted date 26.07.2021

This is an open access article

Published date 30.07.2021 under the Creative Commons CC BY license

How to cite: Sharmazanova, O., Safonova, I., Mityakova, Y. (2021). The importance of compression sonoelastography in improving the diagnostics of the pathology of myometrium. EUREKA: Health Sciences, 4, 65-72. doi: http://doi.org/10.21303/ 2504-5679.2021.001908 